\title{
A 3-mirror surface force balance for the investigation of fluids confined to nanoscale films between two ultra-smooth polarizable electrodes
}

Cite as: Rev. Sci. Instrum. 89, 123901 (2018); https://doi.org/10.1063/1.5045485

Submitted: 20 June 2018 . Accepted: 11 November 2018 . Published Online: 10 December 2018

C. D. van Engers, M. Balabajew, A. Southam, and S. Perkin
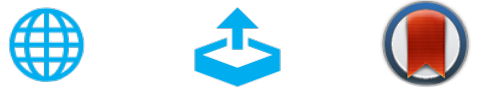

\section{ARTICLES YOU MAY BE INTERESTED IN}

Multiple-beam optical interferometry of anisotropic soft materials nanoconfined with the surface force apparatus

Review of Scientific Instruments 89, 085112 (2018); https://doi.org/10.1063/1.5038951

Structure and dynamics of mica-confined films of $\left[\mathrm{C}_{10} \mathrm{C}_{7}\right.$ Pyrr] $[\mathrm{NTf} 2]$ ionic liquid

The Journal of Chemical Physics 148, 193808 (2018); https://doi.org/10.1063/1.5007809

Contact-free calibration of an asymmetric multi-layer interferometer for the surface force balance

Review of Scientific Instruments 88, 123903 (2017); https://doi.org/10.1063/1.5006056

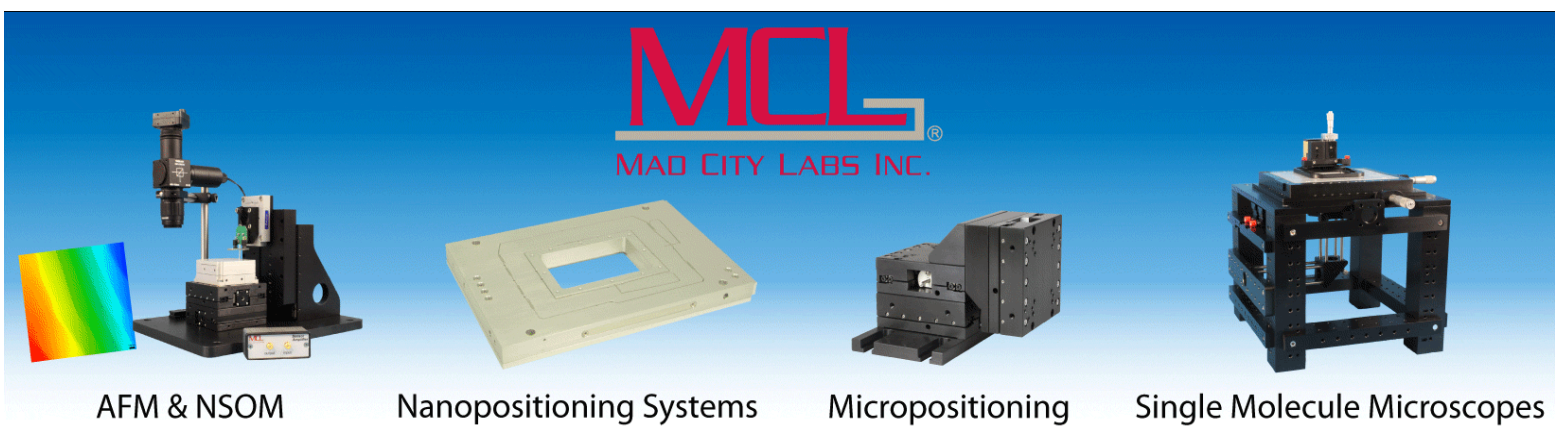




\title{
A 3-mirror surface force balance for the investigation of fluids confined to nanoscale films between two ultra-smooth polarizable electrodes
}

\author{
C. D. van Engers, ${ }^{\text {a) }}$ M. Balabajew, ${ }^{\text {a) }}$ A. Southam, and S. Perkin ${ }^{\text {b) }}$ \\ Physical and Theoretical Chemistry Laboratory, Chemistry Department, University of Oxford, South Parks Road, \\ Oxford OX1 3QZ, United Kingdom
}

(Received 20 June 2018; accepted 11 November 2018; published online 10 December 2018)

\begin{abstract}
We present a new technique, based on the Surface Force Balance (SFB), for the direct measurement of surface forces between two ultra-smooth and polarizable gold electrode surfaces across thin fluid films. Combining the direct interferometric measurement of surface separation and contact geometry with smooth electrode surfaces has proved challenging in the past, and for this reason, previous measurements with the SFB typically involved two insulating mica surfaces, or one mica surface and one electrode surface, or an alternative less direct measure of the surface separation. Here, we demonstrate that a 3-mirror interferometer can overcome these difficulties: the setup involves two ultra-smooth electrode/mirror surfaces between which the fluid is confined and a third mirror to allow for interferometric detection of the liquid thickness with nanometer resolution and at thicknesses much smaller than the diffraction limit of the light. We conclude with a proof-of-concept measurement across dry nitrogen gas. The technique should prove useful for studying the properties of fluids confined at the nanoscale inside a slit-pore of controlled electrical potential or subject to applied electric fields. Published by AIP Publishing. https://doi.org/10.1063/1.5045485
\end{abstract}

\section{INTRODUCTION}

The properties of electrolytes at electrode-electrolyte interfaces and in confined geometries are of fundamental importance in the successful operation and improvement of many current and future technologies, from supercapacitors to dye-sensitized solar cells or polymer-electrolyte batteries and fuel cells.

The Surface Force Balance (SFB, also known as "Surface Force Apparatus") has provided pioneering measurements of the electrochemical double-layer, including structural and frictional forces between surfaces in dilute ${ }^{1-3}$ and more concentrated electrolytes, ${ }^{4,5}$ providing a wealth of knowledge into the nature of the solid-electrolyte interface. However, whilst experiments in many different liquids have been performed, these studies have predominantly used the same solid: muscovite mica.

Mica is a transparent, crystalline, layered aluminosilicate mineral that can be cleaved to reveal atomically smooth single crystal facets of order $\sim \mathrm{cm}^{2}$ area. ${ }^{6}$ The large area atomic smoothness and the optical transparency of the mica surface have made it a favoured material among SFB users. During SFB measurements, two equally thick (i.e., from the same facet), back-silvered mica sheets are placed face-to-face in cross-cylindrical geometry (equivalent to that of a sphere on a flat surface), and the distance between them is measured using white light interferometry. ${ }^{7}$ The mica serves both as an ultrasmooth substrate and interferometric spacer so that even at surface separations much smaller than the diffraction limit of light, the distance between the silver mirrors is large enough

a) C. D. van Engers and M. Balabajew contributed equally to this work.

b) Author to whom correspondence should be addressed: susan.perkin@ chem.ox.ac.uk to allow for the observation of interference fringes. The use of such transparent mica spacers allows for direct determination of the surface geometry and for the measure of refractive index changes in the medium of interest. ${ }^{8}$

However, mica is an insulator and therefore its surface electrical potential cannot be controlled. By contrast, many modern scientific questions, for example, concerning the behavior of electrolytes in energy storage devices or even biological membranes, concern surface potential changes.

To extend the range of experiments that can be performed, a variety of SFB-techniques using polarizable materials, rather than mica, have been explored. The simplest approach is to modify one of the lenses, replacing one of the mica crystals with a thin, transparent layer of template stripped gold, thus using an asymmetric mica-gold setup. Template stripping involves physical vapor deposition (PVD) of a metal on a smooth template. Due to the poor adhesion between the template and the metal, the metal can be glued to another substrate and then peeled off from the template. This reveals a smooth metal surface, which can be an order of magnitude smoother than if deposited directly. ${ }^{9}$ Gold surfaces prepared by template stripping can have RMS roughnesses down to $0.2 \mathrm{~nm} .{ }^{10}$ This is much rougher than the atomically smooth surface of mica $(0.034 \mathrm{~nm} \text { RMS })^{11}$ but still smaller than the length scales of many forces studied in electrolytes. The low roughness combined with the optical properties and electrochemical stability of gold thus enables the measurement of surface forces with small length scales, such as the electrostatic screening length in electrolytes. ${ }^{12}$

Various groups have employed the asymmetric gold-mica setup to study the interaction between gold and mica in dilute electrolytes, ${ }^{13,14}$ such as the first observation of charge inversion between such surfaces. ${ }^{15}$ Other asymmetric setups have been proposed using mica and a $\mathrm{Hg}$ droplet $^{16}$ or mica and 
graphene. ${ }^{17}$ However, the asymmetry and inability to directly measure or control the electrical potential of the mica surface limit the type of experiments that can be performed and pose challenges to the interpretation of the experimental results.

Several symmetric setups have been proposed. ${ }^{18-20}$ The twin-path surface force apparatus, developed by the group of Kurihara in 2008, allows for the use of two smooth opaque substrates, such as thick, template stripped metal films. ${ }^{20,21}$ In this setup, distances are not measured by measuring the constructive interference of light between the metal surfaces. Rather, laser interferometry is used to determine the displacement of a mirror situated at the back of one of the lenses. A drawback of this approach is the loss of optical information. Since light does not pass through the studied medium or interfaces, there is no direct measure of changes to the refractive indexes or the surface geometry, such as the radius of curvature or continuity of the metal film. The latter is particularly important when using metal surfaces, which may be damaged when brought into contact, due to cold welding. Furthermore, this approach requires significant device modification.

An alternative approach was proposed by Levins and Vanderlick and relies on having one template stripped metal surface on one lens and a doubly mirrored mica crystal on the other. ${ }^{19}$ In this 3-mirror approach, optical information regarding the surface and medium is maintained and no modifications to the SFB are required. However, the metal film on the mica cannot be prepared via template stripping and must be deposited directly. It therefore presents a rough surface with a RMS roughness $>1 \mathrm{~nm}$, which makes the technique unsuitable for the measurement of surface forces with small length scales.
We recently developed a simple technique for the fabrication of uniform epoxy layers, amenable to producing a 3-mirror setup in which both metal surfaces are fabricated via template stripping. ${ }^{22}$ Here, we present a detailed account of the preparation and operation of a 3-mirror SFB having two template stripped gold surfaces. This setup allows for easily accessible surface force measurements across confined electrolytes with potential control of both ultra-smooth electrode surfaces.

First, we will describe the setup and provide an interpretation of the obtained interferometric data. Important system parameters and experimental considerations are described in detail. We will discuss the experimental procedure and analysis and demonstrate the proof-of-concept measurement of van der Waals forces between gold surfaces in dry nitrogen.

\section{BACKGROUND}

\section{A. Description of the 3-mirror SFB setup}

Figure 1(a) shows a schematic of a 3-mirror SFB setup using gold as material for the mirrors. Both surfaces are coated with template stripped gold (Au2 and Au3) and supported by a layer of epoxy sitting on top of a cylindrical SFB lens. Figure 1(b) shows a Contact Mode Atomic Force Microscope (CM-AFM) height map of a template stripped gold surface, taken over a $5 \times 5 \mu \mathrm{m}^{2}$ area. The measured RMS roughness is $0.23 \mathrm{~nm}$, much smoother than for surfaces prepared by direct PVD. ${ }^{9,10}$ The cylindrical lenses are placed in crossed-cylinder configuration, which is geometrically equivalent to a sphere on a flat surface and ensures a single point of contact with well-defined geometry. Light enters from the bottom and
A

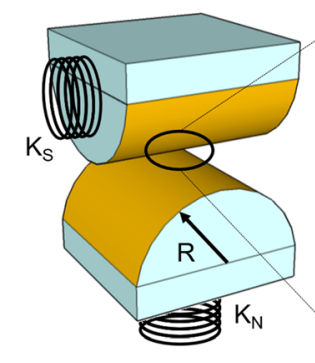

B

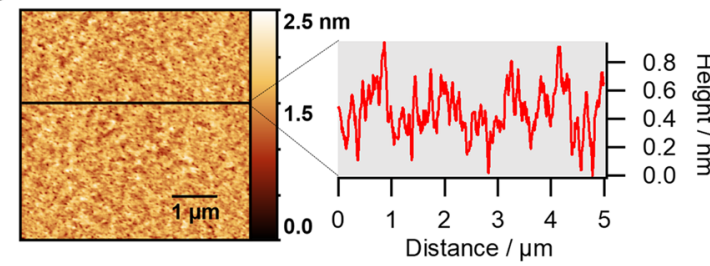

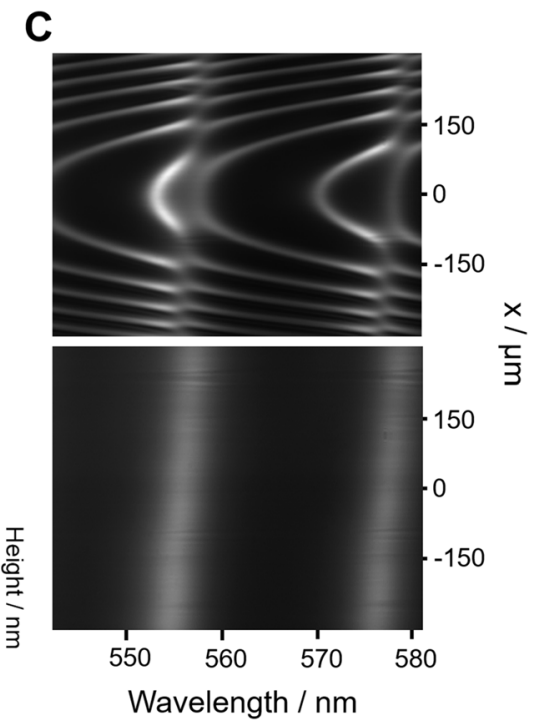

FIG. 1. (a) (Left) Schematic showing the 3-mirror SFB setup using gold as the mirror material. The gold coated lenses are placed in crossed-cylinder configuration, geometrically equal to a sphere on a flat surface. Normal and lateral forces are measured by monitoring the displacement of the surfaces mounted on springs with known spring constant $\left(\mathrm{K}_{\mathrm{S}}\right.$ and $\left.\mathrm{K}_{\mathrm{N}}\right)$ with respect to a known applied motion. (Right) Light enters the setup, constructively interferes between the different mirrors, and emerges as an interference pattern. Primary fringes $[\mathbf{P}(\mathbf{D})$, shown in green] depend on the distance between the mirrors $(\mathbf{D})$ and originate from reflections between mirrors Au2 and Au3, across the medium. Secondary fringes (S, shown in red) are distance independent and arise from reflections between mirrors Au1 and Au2, across the epoxy spacer with thickness Z1. Distance dependent tertiary fringes [T(D), shown in blue] arise from reflections between mirrors Au1 and Au3. (b) CM-AFM height map of a template stripped gold surface. The measured RMS roughness is $0.23 \mathrm{~nm}$. The height profile shown on the right corresponds to the line drawn across the image. (c) Experimental interferograms. In the upper panel, the primary fringes are visible as curved arcs. The apex of the primary fringes indicates the point of closest approach between the surfaces. Secondary fringes are visible as vertical bands superimposed on the primary fringes. The lower panel shows an interferogram recorded whilst rapidly changing the surface separation, $\mathbf{D}$, in order to average the $\mathbf{P}(\mathbf{D})$ and $\mathbf{T}(\mathbf{D})$ fringes; as a result, only $\mathbf{S}$ fringes are resolved. The resulting interferogram shows only the distance independent secondary fringes and corresponds to the thickness $\mathbf{Z 1}$. 
emerges from the other end if it interferes constructively due to the reflections between the mirrors. To be able to measure distances when the separation between the surfaces $\mathbf{D}$ is smaller than the diffraction limit of light, an additional gold layer (Au1) is present underneath one of the template stripped surfaces, separated by epoxy of thickness $\mathbf{Z 1}$.

Three different reflections are identified and are expected to give rise to different FECO patterns in the interferogram. Distance dependent primary fringes $(\mathbf{P}(\mathbf{D}))$, marked in green, result from reflections between the mirrors on the surfaces in contact with the medium, Au2 and Au3. A stationary set of secondary fringes ( $\mathbf{S}$, shown in red) results from reflections between Au1 and Au2. Finally, a third set of distance dependent tertiary fringes arise [T(D), shown in blue] from reflections between the gold coating on the lens and that on the other surface, between Au1 and Au3.

Figure 1(c) shows the experimental interferograms of the 3-mirror setup taken at a large surface separation. The upper panel shows an image taken with the surfaces at rest. The curved bands represent the primary fringes, $\mathbf{P}(\mathbf{D})$. The apex of the primary fringes represents the point of closest approach between the surfaces Au2 and Au3. The secondary fringes, $\mathbf{S}$, are visible as two approximately vertical bands. By recording an interferogram whilst rapidly changing the surface separation, the distance dependent fringe contributions can be averaged out. The lower panel in Fig. 1(c) shows such an interferogram in which only the secondary fringes are visible. Owing to the special preparation method used to glue down the Au2 layer for template stripping, the thickness of the epoxy layer, Epoxy1, is virtually constant in the region of closest approach as indicated by the straight and close-tovertical profile of $\mathbf{S}$. The thickness uniformity of the Epoxy1 is crucial for performing experiments as it allows for reliable interpretation of interference spectra and ensures a single point of contact between the surfaces. The tertiary fringes cannot clearly be distinguished, as we next discuss.

\section{B. Interpretation of the interference pattern}

In order to rationalise the absence of apparent tertiary fringes, it is informative first to consider a simple approximation, in which light intensity $I$ of each fringe pattern is the product of light that passes through the interferometer through different transmissions and reflections. The transmission coefficient is $T_{i, j}$ and the reflection coefficient is $R_{i, j}$, where $\mathrm{i}, \mathrm{j}$ refers to the materials at the reflecting interface. Approximating the mirrors to be much thinner than the wavelength of light and non-absorbing and using the geometry shown in Fig. 1, it follows that for the different fringe orders

$$
\begin{gathered}
I_{P(D)} \sim T_{A u, \text { Lens }} T_{A u, \text { Epoxy }}^{3} T_{A u, \text { Medium }}^{2} R_{A u, \text { Medium }}^{2}, \\
I_{S} \sim T_{A u, \text { Lens }} T_{A u, \text { Epoxy }}^{3} T_{A u, \text { Medium }}^{2} R_{A u, \text { Epoxy }}^{2}, \\
I_{T(D)} \sim T_{A u, \text { Lens }} T_{A u, \text { Epoxy }}^{5} T_{A u, \text { Medium }}^{4} R_{A u, \text { Medium }} R_{A u, \text { Epoxy }} .
\end{gathered}
$$

Equations (1)-(3) illustrate that compared to the primary and secondary fringes $\left[I_{P(D)}\right.$ and $\left.I_{S}\right]$, the light intensity of the tertiary fringes $\left(I_{T(D)}\right)$ results from two additional transmissions through the Au-Epoxy and Au-Medium interfaces $\left(T_{A u, E p o x y}\right.$ and $\left.T_{A u, \text { Medium }}\right)$. According to Fresnel's equations for light at normal incidence to the surfaces, ignoring the absorbance or dispersion of light in the gold layer and using $T_{A u, \text { Epoxy }} \approx 0.55$ and $T_{\text {Au,Medium }} \approx 0.71$ (in case the medium is air), $I_{T(D)} / I_{S, P(D)}$ $\approx 0.15$. The tertiary fringes are thus expected to be lower in intensity than the primary and secondary ones.

To understand the effect of tertiary fringes in the interference spectra, different mirror configurations were calculated using the multilayer matrix method, described in detail elsewhere. $^{23-25}$ A comparison between different mirror configurations is shown in Fig. 2. The first scenario considered, in Figs. 2(a)-2(c), is the classic 2-mirror SFB setup indicated in the schematic in panel (c). Panel (a) shows an intensity plot indicating the fringe position as a function of distance for this classic 2-mirror setup for which the thickness of Au2 $=0 \mathrm{~nm}$
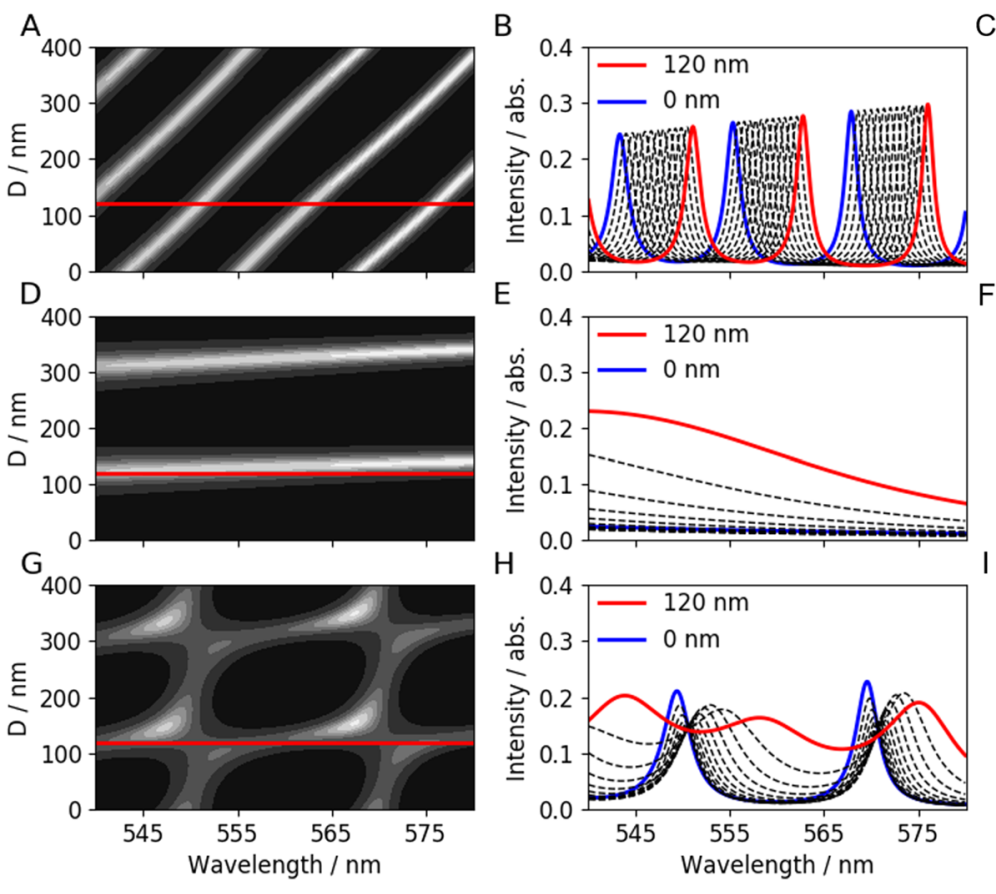

F
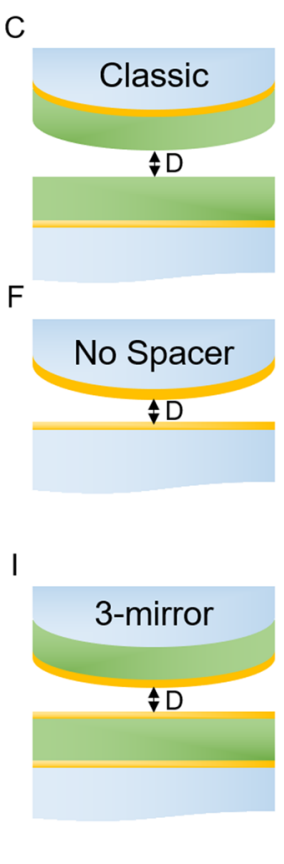

FIG. 2. Calculated dependence of interference fringes on surface separation $\mathbf{D}$ for different mirror configurations. [(a)-(c)] Conventional 2-mirror SFA setup. [(d)-(f)] 2-mirror setup in the absence of spacer layers. [(g)-(i)] 3-mirror setup. The left-most panel shows an intensity plot of the interference fringes as a function of wavelength and surface separation. The red line drawn at $\mathbf{D}=120 \mathrm{~nm}$ corresponds to the red interference spectrum in the central panels. The central panels show the progression of a single interference spectrum from $\mathbf{D}=120 \mathrm{~nm}$ (red solid line) to $\mathbf{D}=0 \mathrm{~nm}$ (blue solid line) with $10 \mathrm{~nm}$ steps (black dashed lines). The right panels show a schematic of the different configurations. 
and the thicknesses of the epoxies $\mathbf{Z 1}=\mathbf{Z 2}=4 \mu \mathrm{m}$, using refractive indexes $\mathrm{n}_{\text {Epoxy }}=1.55$ and $\mathrm{n}_{\text {Medium }}=1.45$. The horizontal red line in A indicates $\mathbf{D}=120 \mathrm{~nm}$; the interference spectra corresponding to $\mathbf{D}$ decreasing from $120 \mathrm{~nm}$ (solid red line) to contact (solid blue line) in $10 \mathrm{~nm}$ steps (black dots) are shown in panel (b). The calculations show that for the 2-mirror setup, the fringes have a clear shape and move nearly linearly across the range examined as $\mathbf{D}$ is decreased.

A second scenario, considered in Figs. 2(d)-2(f), is of two mirrors on the face of the lenses but no third mirror, i.e., the absence of a spacer layer. A broad interference fringe can only be observed at specific distances and completely disappears close to contact, emphasising the need for a spacer with an optical thickness larger than the diffraction limit.

The third calculated scenario is of our proposed 3-mirror setup, using $\mathbf{Z 1}=4.9 \mu \mathrm{m}, \mathbf{A u 1}=30 \mathrm{~nm}, \mathbf{A u 2}=20 \mathrm{~nm}$, and Au3 $=30 \mathrm{~nm}$, shown in Figs. 2(g)-2(i). The intensity plot [panel (g)] shows two horizontal bands corresponding to the primary fringes, similar to the situation shown in Fig. 2(d), and a vertical set of two secondary fringes. It is apparent that, as $\mathbf{D}$ is decreased (increased), the tertiary reflections are responsible for the blue-shift (red-shift) of the interference fringes.

To verify this interpretation, additional calculations for different mirror geometries were performed. Figure 3(a) shows the expected intensity plot for interference spectra as a function of distance and wavelength when Au1 $=\mathbf{A u} 2=40 \mathrm{~nm}$ and Au3 $=0 \mathrm{~nm}$. This configuration shows the characteristic distance independent secondary fringes, which appear as vertical bands in the plot and are highlighted by the dashed red line. Another extreme configuration is shown in Fig. 3(d), in which Au2 = Au3 = $40 \mathrm{~nm}$ and Au1 = $0 \mathrm{~nm}$. Here, only the distance dependent primary fringes are shown and highlighted by the green dotted line. The effect of the tertiary reflections becomes apparent when examining Figs. 3(b) and 3(c), for which Au1 = Au3 = $40 \mathrm{~nm}$ and $\mathbf{A u 2}=10 \mathrm{~nm}$ [in (b)] and $\mathbf{A u 2}=40 \mathrm{~nm}$ [in (c)]. These intensity plots verify the simple result from Eqs. (1)-(3): the presence and influence of the tertiary fringes on the interference spectra become more pronounced as the transmission through the Au-Epoxy and Au-Medium interfaces becomes higher. Indeed, for Au2 = $40 \mathrm{~nm}$ [Fig. 3(c)], the blueshift caused by the tertiary fringes is almost negligible, whereas for Au2 $=10 \mathrm{~nm}$ [Fig. 3(b)], the blueshift caused by the tertiary fringes is so pronounced that the intensity plot begins to resemble that of the classic two-mirror SFB [Fig. 2(a)].

\section{Experimental considerations}

We have seen in Fig. 3 that the thickness of the gold layers directly impacts spectral resolution and therefore distance resolution of the setup. In the conventional SFB-setup, resolution in surface separation is easily smaller than $1 \mathrm{~nm}$ and can optimally reach $0.1 \mathrm{~nm}$. It is therefore important to consider the system parameters for our proposed three mirror setup, such as Au layer thicknesses and spacer thickness, to optimise resolution. In Fig. 4, we show the calculated changes in the peak position of the left-most peak for different mirror and spacer thicknesses, calculated from a surface separation of $\mathbf{D}=100 \mathrm{~nm}$ down to contact. The spectral resolution is optimal when the central gold thickness (Au2) is minimal and the spacer thickness is minimal but larger than the diffraction limit of light. However, reducing these parameters has important implications for the analysis of the interferometric data and practical realisation of the setup.

When two (sufficiently clean) gold surfaces are brought into contact in vacuum or air, they can form a so-called "cold weld" due to strong adhesion. Upon separation, this high adhesion causes damage to the surfaces. Indeed, in the 3-mirror setup presented here, for which the contact area between the surfaces is much larger than the thickness of the gold films, one layer may simply be transferred onto the other, as shown in Fig. 8 (Appendix). Due to this phenomenon, determining the thickness of $\mathbf{Z 1}$ by performing a so-called "contact calibration,", as is performed for the conventional 2-mirror SFB setup which uses black-silvered mica rather than gold [see Fig. 2(g)], is not possible. However, due to the brightness
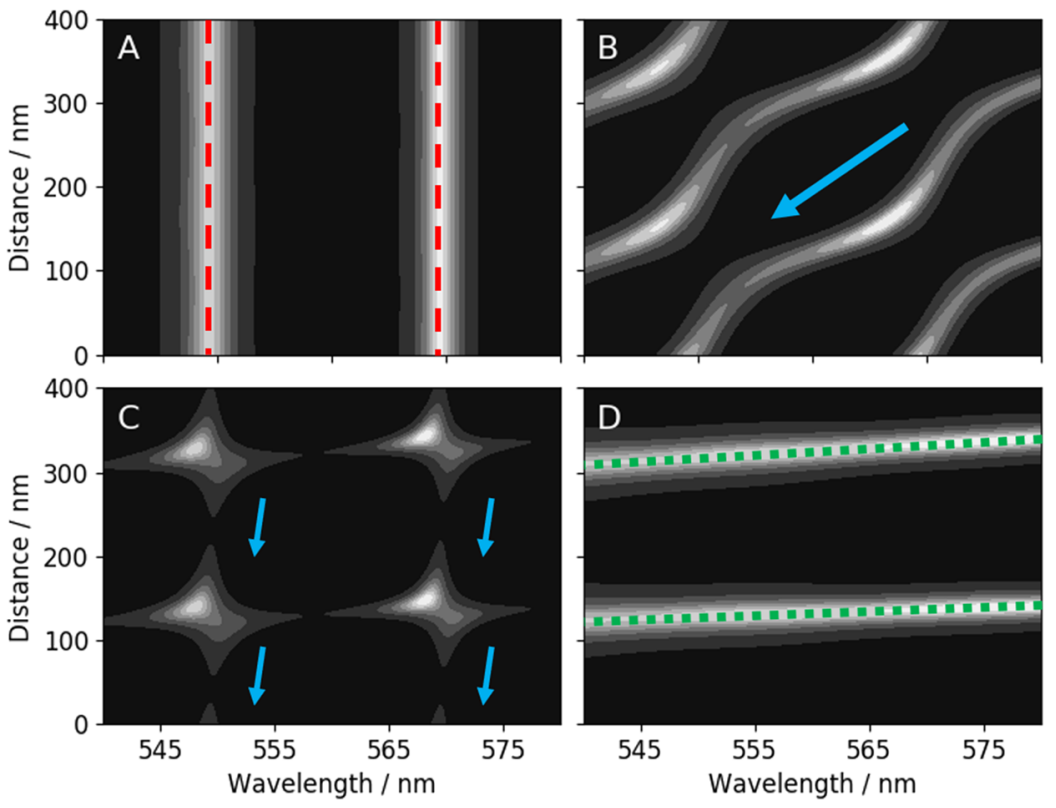

FIG. 3. Calculated fringe intensity as a function of wavelength and distance for the 3-mirror SFB setup using different gold thicknesses. (a) Au1 $=\mathbf{A u 2}=40 \mathrm{~nm}$ and Au3 $=0 \mathrm{~nm}$. Due to the absence of mirror Au3, only the distance independent secondary fringes, which result from reflections between mirrors Au1 and Au2, are visible. (b) Au1 = $40 \mathrm{~nm}, \mathbf{A u 2}=10 \mathrm{~nm}, \mathbf{A u 3}=40 \mathrm{~nm}$. The distinction between different sets of primary, secondary, or tertiary fringes cannot readily be made. Since Au2 is thin with respect to the other two mirrors, the effect of the tertiary fringes is relatively strong and the calculated fringe intensity starts to resemble that of the classic 2-mirror SFB. (c) Au1 = $40 \mathrm{~nm}, \mathbf{A u 2}=40 \mathrm{~nm}, \mathbf{A u 3}$ $=40 \mathrm{~nm}$. Since Au2 is now equal in thickness to the other two mirrors, the distance dependent shift in fringe intensity due to the tertiary fringes is much less pronounced than in (b). (d) Au1 $=0 \mathrm{~nm}, \mathbf{A u 2}=40 \mathrm{~nm}$, Au3 $=40 \mathrm{~nm}$, corresponding to a 2-mirror interferometer without a spacer layer, equivalent to the situation shown in Figs. 2(d)-2(f). 

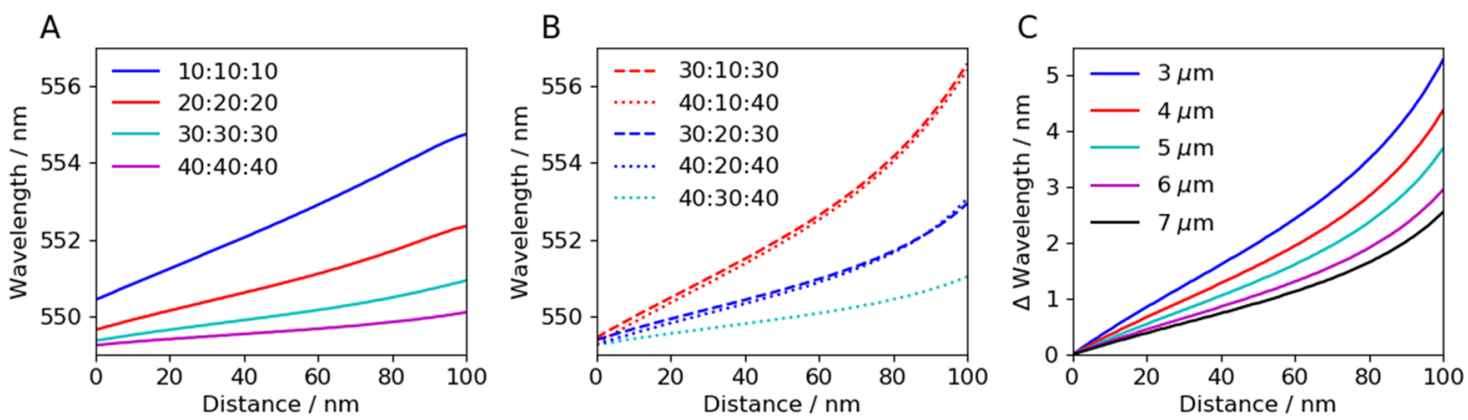

FIG. 4. Calculated effect of the mirror configuration on spectral resolution. The wavelength shift of the leftmost peak in the wavelength range $540-580 \mathrm{~nm}$ upon decreasing the surface separation from $\mathbf{D}=100 \mathrm{~nm}$ to contact is shown as a function of gold thickness, using $\mathbf{Z 1}=4.9 \mu \mathrm{m}$ in (a) and (b) and as a function of spacer thickness in (c), using Au1 $=30 \mathrm{~nm}, \mathbf{A u 2}=20 \mathrm{~nm}, \mathbf{A u 3}=30 \mathrm{~nm}$. The gold and epoxy thicknesses used in the calculation are shown in the legends.

of the secondary fringes, the thickness of $\mathbf{Z 1}$ can readily be determined by fitting the secondary fringe profile.

It is clear from Fig. 3 that the position of the secondary fringes may be strongly influenced by the presence of the distance dependent fringes $\mathbf{P}(\mathbf{D})$ and $\mathbf{T}(\mathbf{D})$. However, due to their distance dependence, their effect may be "averaged out": experimentally this is performed by recording an interferogram whilst moving the surfaces (varying $\mathbf{D}$ ), as has been suggested by Heuberger and shown in our group. ${ }^{24,26}$ Computationally, the secondary fringe profile can be calculated by averaging interference spectra at multiple distances at a large surface separation, analogous to the experiment. The thickness of $\mathbf{Z 1}$ can then be obtained by fitting the averaged experimentally obtained profile to the averaged calculated one. The consequence of this calibration step is that to obtain a reliable fit, at least two secondary fringes should be visible in the wavelength range of interest, which puts a lower limit on the value of $\mathbf{Z 1}$. The ideal thickness of the epoxy spacer in our setup is then 4-5 $\mu \mathrm{m}$ (see Fig. 9 in the Appendix).

It is important to note that we here assume that the thickness $\mathbf{Z 1}$ has a constant value at the point of closest approach of the crossed-cylinder lenses, allowing us to use the calibrated value in the subsequent calculation of $\mathbf{D}$ during a force run. In an ideal experiment where the light is aligned perfectly perpendicular to the contact point and where surfaces translate toward each other in a perfectly axial way, $\mathbf{Z 1}$ will indeed be a constant during the surface approach. However small misalignments of the optical or mechanical axis can cause small lateral shifts of the contact point during the approach, and therefore-since the epoxy is not of precisely uniform thickness across the lens-it is necessary to consider changes in $\mathbf{Z 1}$ during the approach. In our present experiments using the previously developed methodology for creating ultra-smooth and uniform epoxy films, we calculate from the typical lateral gradients of the secondary fringes [such as in Fig. 1(c)] a thickness variation of below $0.2 \mathrm{~nm}$. In this case, the impact on calculation of $\mathbf{D}$ is negligible. However if the epoxy layer were less uniform, then a correction for this would be necessary in the processing of force profiles.

Another important consideration is the experimentally achievable thickness of the gold layers. For the fabrication of template stripped gold surfaces onto curved substrates, such as the cylindrical lenses used here, mica may be used as a template. ${ }^{10}$ Here, the growth mode of the gold film during deposition determines the minimal gold thickness that can be obtained since the ability to template strip relies on the difference in adhesion and contact area at the gold-mica and the gold-epoxy interfaces. We found that using a simple, thermal evaporation approach, a minimal gold thickness of $20 \mathrm{~nm}$ ensured the transfer of an ultra-smooth, conformal film [Fig. 1(b)].

The use of secondary fringe patterns for the determination of the thickness of $\mathbf{Z 1}$ and minimal gold thickness used $(\sim 20 \mathrm{~nm})$ has important implications for the data analysis. First, fitting the full spectrum of the obtained averaged secondary fringes data gives a more reliable fit. Second, when using a thickness of $\mathbf{A u 2}=20 \mathrm{~nm}$ for the middle, template stripped gold layer, changing the distance between the surfaces causes the interference spectra to evolve such that simple tracking of the fringes is no longer possible. We therefore fit the experimentally obtained interference spectra in the entire spectral range, rather than fitting the peak positions as conventionally performed. ${ }^{25}$

Finally, in order to fit the experimental data, it is important to match the refractive index of the epoxy to that of the underlying lens. When the Epoxy2 layer has a different refractive index than the underlying lens, this results in an additional reflection at the Epoxy2-lens interface. The effect of such an index mismatch using $\mathrm{n}_{\text {Epoxy } 2}=1.55, \mathrm{n}_{\text {Lens }}=1.46, \mathbf{D}=0$, and $\mathbf{Z 1}=4.9 \mu \mathrm{m}$, as a function of the thickness of the Epoxy2 layer is shown in Fig. 5. Figure 5(a) shows that the intensity ratio between the two visible peaks changes significantly when the thickness of the Epoxy2 layer is changed only $90 \mathrm{~nm}$. Furthermore, Fig. 5(b) shows how the peak intensity ratio does not change linearly but is very sensitive to changes in the thickness of the Epoxy2 layer with respect to the thickness $\mathbf{Z 1}$ (the Epoxy1 layer). When the epoxy and underlying lens are index matched, i.e., $\mathrm{n}_{\text {Epoxy } 2}=\mathrm{n}_{\text {Lens }}$, the variance in peak intensity disappears [black lines Fig. 5(b)]. Thus to avoid having to introduce the thickness of this layer as an additional fitting parameter, matching the refractive index of the epoxy to that of the underlying lens is highly desirable.

In our particular setup, we find that gold thicknesses of Au1:Au2:Au3 = 35:20:35 nm in combination with a spacer thickness of 4-5 $\mu \mathrm{m}$ (for $\mathrm{n}_{\text {Spacer }}=1.55$ ) present optimal system parameters and can be easily realized in experiment. We find that the minimal thickness of the central (template stripped) 

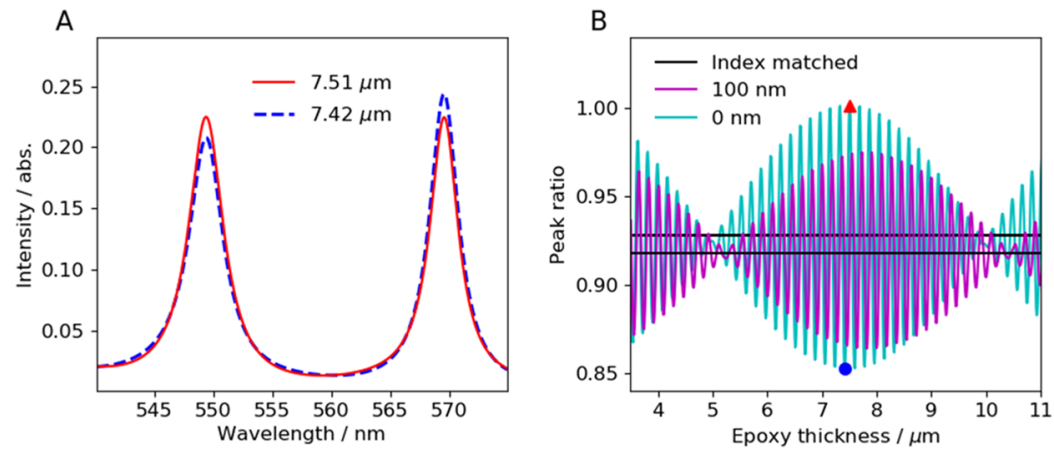

FIG. 5. Effect of the refractive index mismatch between the epoxy supporting Au3 layer (Epoxy2) and the underlying glass lenses. (a) Difference in peak intensities for two different thicknesses of the layer Epoxy2, using $n_{\text {Epoxy } 2}=1.55$ and $n_{\text {lens }}=1.46$. (b) Thickness dependence of the intensity ratio between the two peaks visible in the 540-580 nm wavelength range, as a function of the thickness of the Epoxy2 layer. The index mismatched case, using $\mathrm{n}_{\mathrm{Epoxy} 2}=1.55$ and $\mathrm{n}_{\text {lens }}=1.46$, is shown in turquoise (for $\mathbf{D}=0 \mathrm{~nm}$ ) and purple (for $\mathbf{D}=100 \mathrm{~nm}$ ). When the refractive index of the lens and the epoxy are the same, the fringe intensity is no longer influenced by the thickness of the Epoxy2 layer (black straight lines for both $\mathbf{D}=0 \mathrm{~nm}$ and $\mathbf{D}=100 \mathrm{~nm}$ ).

gold layer required to obtain a conformal gold film that can be template stripped is $\sim 20 \mathrm{~nm}$.

\section{MATERIALS AND METHODS}

\section{A. Experimental setup and lens preparation}

Experiments were performed using the surface force balance as described by Kumacheva and Klein. ${ }^{27}$ The interference fringes were recorded with a charge-coupled device (CCD; Retiga R6, QImaging). Emission lines from a mercury vapour lamp were recorded and used to convert the recorded fringe positions from pixel to wavelength. All mica and lenses were handled in Bassaire Limited P3HF laminar flow hoods (LFH). All glassware and lenses were cleaned in piranha baths [concentrated sulfuric acid (99.999\%) and hydrogen peroxide, 3:1]; all metallic tools were cleaned in aqueous nitric acid solution unless indicated otherwise.

Hemi-cylindrical N-BaLF5 lenses (radius of curvature $=$ $1 \mathrm{~cm}$ ) were obtained from Scientific Optical. N-BaLF5 lenses were chosen in order to match the refractive index of the EPON $1004 \mathrm{~F}$ epoxy glue (Shell, $\mathrm{n}=1.544 \pm 0.025$ as measured by ellipsometry), used to support the template stripped gold layers (Fig. 5). One of the lenses has two gold coatings [Au1 and Au2, see Fig. 1(a)]. Au1, which rests directly on the lens, was prepared by thermal evaporation of $2 \mathrm{~nm}$ chromium (as adhesion layer) and $35 \mathrm{~nm}$ gold (Edwards Auto 306 Cryo Evaporator).

The mirrors Au2 and Au3 were prepared via template stripping. First, gold was prepared on a mica template as follows. Muscovite mica (Ruby Mica Grade \#1 V-1/V-2, S\&J Trading, Inc.), to be used as the ultra-smooth template, was cleaved to obtain thin, flexible, and single-facetted pieces with a thickness of 3-8 $\mu \mathrm{m}$. These were placed on another thick and rigid, freshly cleaved base sheet. The single-facetted pieces serve as templates, whereas the base sheet serves as support. ${ }^{10}$ The exposed side of the mica templates was coated in gold (Heraeus; $1 \mathrm{~g}$; fineness 999.9) via thermal evaporation [HHV Auto306 (HVV Ltd.)].

Second, the epoxy glue was prepared by template stripping to provide a smooth glue surface: single-facetted pieces of muscovite mica were cleaved and placed on small silicon chips. These were spin-coated (Ossila Spin Coater; 3000 rpm; $30 \mathrm{~s})$ with a solution of epoxy in ethyl acetate $(0.33 \mathrm{~g} / \mathrm{ml}, 3000$ $\mathrm{rpm}, 30 \mathrm{~s}$ ) and applied epoxy-side down to the lenses heated above the glass transition temperature of the epoxy at $150^{\circ} \mathrm{C}$ on a hotplate. After carefully peeling the mica off under ultrapure water (Sartorius arium comfort, TOC $<2 \mathrm{ppb}$, resistivity $=$ $18.2 \mathrm{M} \Omega \mathrm{cm}$ ), the epoxy glue was heated to $150{ }^{\circ} \mathrm{C}$ for $30 \mathrm{~min}$ to remove residual solvent and the lenses were left to cool to room temperature.

Finally, the smooth epoxy-coated lens was coated with the templated smooth gold: The lenses were heated again $\left(150^{\circ} \mathrm{C}\right)$ and the gold-coated mica was applied gold side down before being left to cool again. To reduce the adsorption of airborne contaminants to the template-stripped gold surface, the top mica layer was stripped to reveal the clean and smooth $\mathrm{Au}$ surface as soon as possible before mounting the lenses into the SFB. The roughness (RMS) of the template-stripped gold layers is typically $0.2-0.4 \mathrm{~nm}$ [Fig. 1(b)].

\section{B. Data analysis}

The data analysis is based on the multilayer-matrix method described elsewhere. ${ }^{24,25}$ The full stack of layers, including the N-BaLF5 glass lenses, Cr wetting film, gold mirrors, and epoxy layers, is included in the calculation. That is, the full interferometer calculation includes the following layers: NBaLF-5 | Cr | Au1 | EPON | Au2 | Air | Au3 | EPON I NBaLF-5. Complex refractive index is used for each, although dispersion of the epoxy was found to be of negligible importance in this case and was not included. For all fitting procedures, the sum of squared residuals (SSR) was used as a similarity function. In order to find the global minimum, a brute-force search is employed to minimize the SSR. In contrast to the fast spectral correlation method introduced by Heuberger, ${ }^{25}$ the entire traces are considered during the fitting procedure. All data analysis was performed in Python using Numpy. ${ }^{28}$

Before fitting, all experimental raw traces are prepared in the following manner: Initially, the background intensity of the camera is subtracted. To take the wavelength dependent intensity of the light source into account, the raw data are divided by the intensity of the light source. Since the light source 

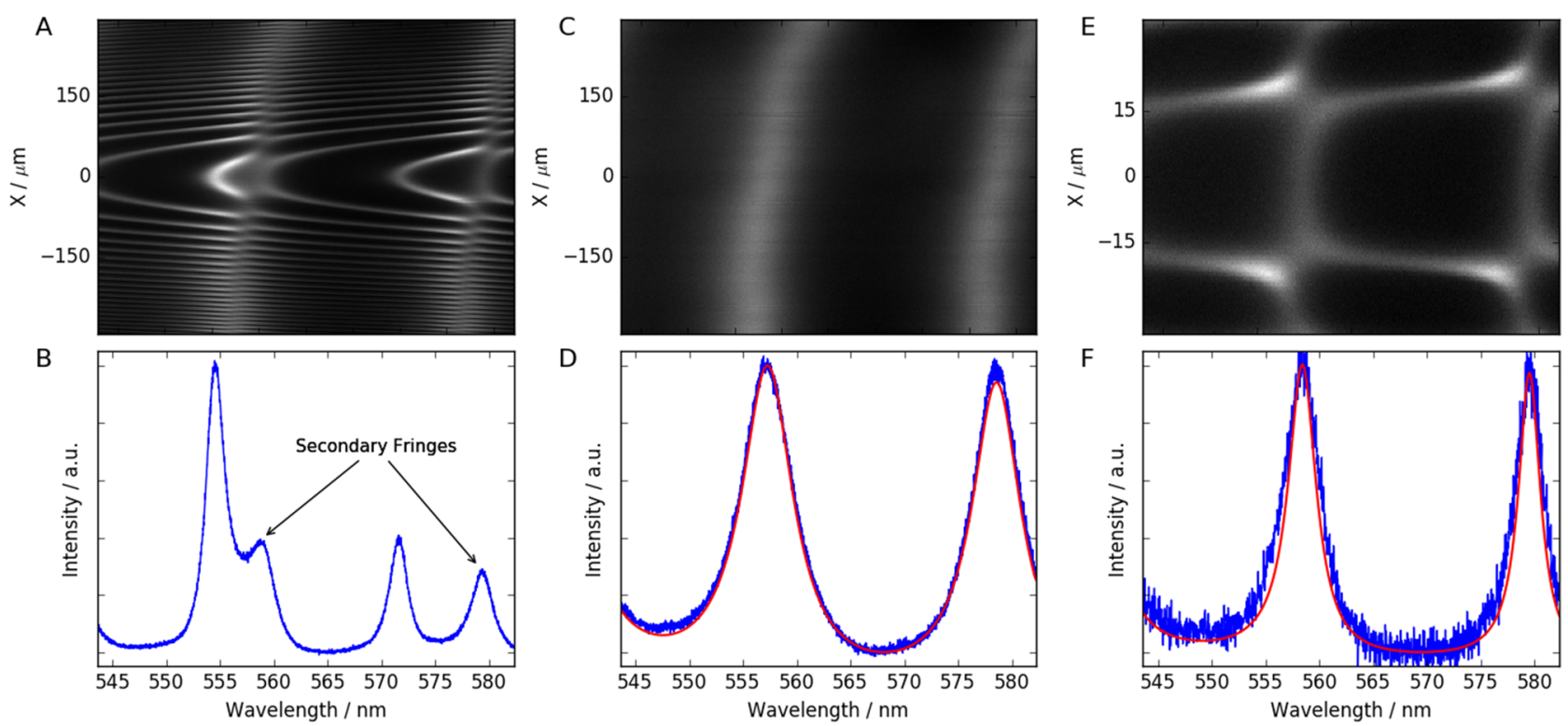

FIG. 6. [(a), (c), and (e)] Images of different interference patterns and [(b), (d), and (f)] corresponding traces (blue lines) with fits (red lines). (a) and (b) show the interference pattern at an intermediate distance. It shows primary fringes as well as secondary fringes. (c) and (d) show solely the secondary fringes obtained by mechanical averaging. The fit (red line) is used to obtain the glue thickness (Z1). (e) and (f) show an interference pattern and the corresponding fit at a distance of approximately $100 \mathrm{~nm}$. The interference pattern consists of secondary fringes which are distorted by tertiary fringes. The wavelength scale (x-axis) of (a), (c), and (e) is equal to the plots below.

can exhibit significant intensity fluctuations, the raw data are then normalized. For normalization, first the minimum intensity of the trace is subtracted setting the background to zero. In a second step, the data are divided by the new maximum intensity. To minimize the influence of noise on the normalization, the minimum and maximum values are obtained from traces which are smoothed by means of a Savitzky-Golay filter.

Analogous to the experimental approach, secondary traces are calculated by averaging over calculated traces for many different gap thicknesses $\mathbf{D}$. To minimize calculation time and reduce noise due to the finite number of calculated spectra, the obtained averaged trace is smoothed by means of a SavitzkyGolay filter.

\section{VALIDATION AND DISCUSSION}

To validate the presented method, we performed an experiment with the proposed lens setup in dry nitrogen. Figure 6(a) shows an image of the measured interference pattern. It shows curved primary fringes similar to those observed in a conventional SFB setup; the curvature in $\mathbf{P}(\mathbf{D})$ fringes tracks the curvature of the SFB lenses in the $\mathrm{x}$-direction. Due to the high reflectivity of the gold layers Au1 and Au2, the secondary fringes are clear with high intensity. This can lead to an irregular appearance of the corresponding fringe pattern [see Fig. 6(b)]. By mechanical averaging, it is possible to collect images without primary fringes [see Fig. 6(c)]. Fitting of the corresponding trace allows for the determination of $\mathbf{Z 1}$, the only free parameter for fitting the secondary fringes. For this particular experiment, $\mathbf{Z 1}=4.79337 \mu \mathrm{m}$.

After determination of $\mathbf{Z 1}$, the surface separation $\mathbf{D}$ is the only free parameter for fitting the experimentally obtained spectrum, which ensures fast and reliable fitting. Figure 6(e) shows a typical interference pattern at a small separation. The corresponding trace [see Fig. 6(f), blue line] can be fitted very well using the known thickness $\mathbf{Z 1}$ resulting in $\mathbf{D}=108.98 \mathrm{~nm}$. This distance is below the diffraction limit of light but can nevertheless be observed with the presented setup.

Figure 7 shows the final evaluated profile, distance $v s$. time, during an approach of the surfaces. Determination of $\mathbf{D}$ is possible for a wide range of separations, even below the diffraction limit of light. From a distance of approximately $100 \mathrm{~nm}$, the surfaces are found to jump to contact due
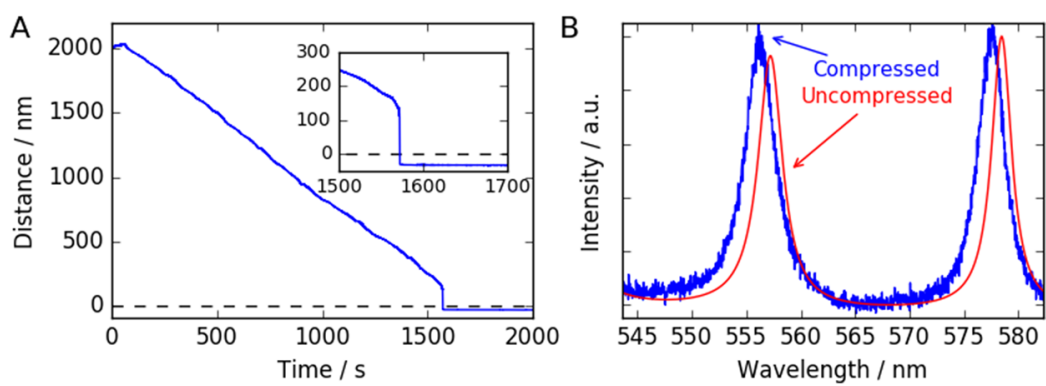

FIG. 7. (a) Approach of surfaces into contact $(\mathbf{D}=0)$. The final position is closer than the contact position expected for the fitted value of $\mathbf{Z 1}$. This is due to the compression of the glue layer. From a distance of approximately $100 \mathrm{~nm}$, the surfaces jump into contact. (b) Fringe pattern of actual contact (blue line) compared to the calculated contact (red line) based on Z1. The difference between the two traces arises from the compression of the epoxy layer upon contact. 
to the strong van der Waals force between the surfaces across gas. This measurement is analogous to the many direct measurements of surface forces across air and liquids performed in the past using the SFB/SFA, now demonstrated to be possible with two ultra-smooth metallic surfaces.

Two details of the measurement, which are particular to our demonstration in nitrogen gas, should be mentioned. First, the resulting contact position occurs at an apparently negative value for $\mathbf{D}$. This unphysical outcome is an artefact of the fitting procedure, which assumes that the epoxy thickness $\mathbf{Z 1}$ is fixed. Due to the compressibility of the glue layer and adhesive forces, the thickness of the epoxy layer is reduced upon contact, resulting in a change in $\mathbf{Z 1}^{29}$ and can mathematically be compensated for by assuming negative values for $\mathbf{D}$. To adjust for this compression, the change in $\mathbf{Z 1}$ can be determined by fixing $\mathbf{D}$ at zero and newly fitting $\mathbf{Z 1}$. (In this demonstration experiment, the glue is initially compressed by $7.5 \mathrm{~nm}$, and during the approach shown, this compression is continuously increased to $9.0 \mathrm{~nm}$.) For a correct description of this compression, a consideration by means of contact mechanics, e.g., based on the Johnson-Kendall-Roberts model of elastic deformation ${ }^{29}$ would be necessary. However, important parameters such as effective Young's modulus for such thin films (which are thinner than the contact diameter) are not known. A detailed discussion of the compression is beyond the scope of this initial description of the method, and for the present demonstration, we estimated a contact diameter approximating the system as a capped sphere of $1 \mathrm{~cm}$ radius on a flat with the cap height corresponding to the compression. For a compression of $9 \mathrm{~nm}$, this equates to a contact diameter of $27 \mu \mathrm{m}$. This is in very good agreement with the contact diameter obtained from the experimental contacting area of the gold surface which is approximately $30 \mu \mathrm{m}$. Notwithstanding this good agreement, the contact mechanics describing the deformation of the epoxy layers in our setup is quite complex and should be addressed in future work; the ability to determine directly the epoxy thickness and contact profile during compression would allow for a detailed study. Although the effect of compression is quite significant in the present demonstration in nitrogen, we note that for an experiment in liquid - the anticipated main application of this techniquethe adhesive forces in the contact zone would be orders of magnitude lower and as such the compression of the spacer layer during measurements in liquids is expected to be substantially less significant. ${ }^{29}$ The second point to note in relation to the validation experiment in air is that bringing two ultrasmooth and clean gold surfaces into contact leads to a so-called "cold weld." Upon separation, the surfaces jump out of contact to distances above $1 \mathrm{~mm}$, which coincides with fracture of the gold film at the periphery of the contact zone and transfer of one of the gold layers (Au2, Au3) to the other (see the Appendix and Fig. 8, where quantitative details of the adhesive force are also considered). Again, this aspect is particular to the demonstration experiment in nitrogen gas and is not expected to occur when the surfaces are separated by liquid. Indeed, in our preliminary experiments with liquids, to be reported in due course, the strong adhesion and surface damage does not occur.

\section{CONCLUSION}

In this work, we presented a 3-mirror surface force balance. The setup allows for the measurement of forces and distances between two template-stripped gold surfaces (RMS roughness $0.2-0.3 \mathrm{~nm}$ ) across liquids. This is achieved by the introduction of a spacer together with an additional gold mirror behind one of the template-stripped surfaces, which ensures that the optical distance between mirrors is sufficiently large to allow for interferometric determination of the surface separation with sub-nm resolution. We presented an analysis of the effect of a 3rd mirror on the interferometric data, followed by analyzing the system parameter space, and provided examples of optimised parameters for experiments. The experiment was demonstrated by performing a measurement in dry nitrogen. In this proof-of-concept measurement, the ability to evaluate the surface separation during an approach from $\sim 10^{3} \mathrm{~nm}$ to contact was demonstrated.

The procedure demonstrated here opens the way to direct measurement of surface forces and properties of liquids confined at the nano-scale between two ultra-smooth polarisable gold electrodes. Advantages compared to previous techniques are that this setup is entirely symmetric, removing the disadvantage of having one ill-defined boundary condition, and both surfaces are ultra-smooth allowing for details to be resolved at the molecular scale. Additionally, the method can be adopted in any standard SFB setup without further modifications.

\section{ACKNOWLEDGMENTS}

The authors acknowledge funding from the European Research Council (under Grant Nos. ERC-2015-StG-676861 and 674979-NANOTRANS), the Leverhulme Trust (Grant No. RPG-2015-328), and the Zvi and Ofra Meitar Magdalen Graduate Scholarship (to C.D.v.E.).

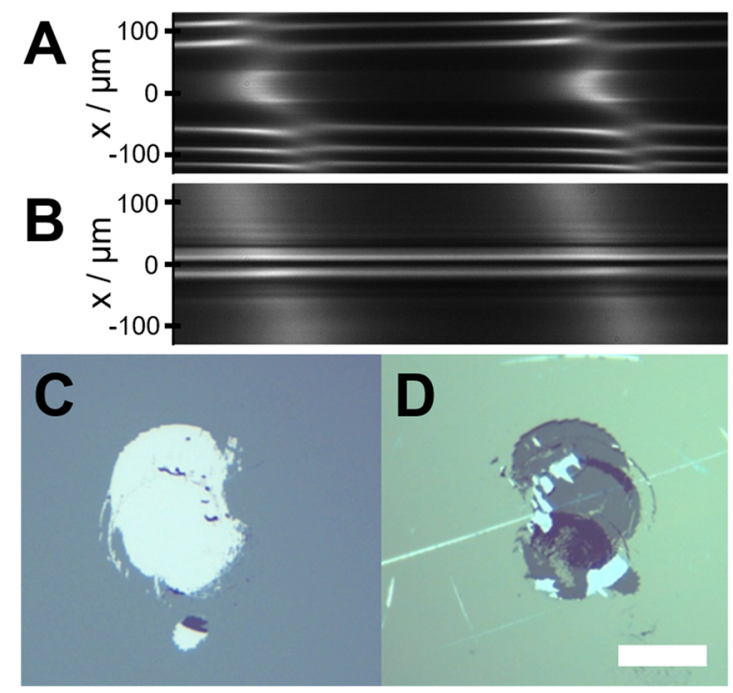

FIG. 8. Effect of cold-welding between gold surfaces Au2 and Au3. When the gold surfaces are brought into contact as shown in the interferogram in (a), they may form a so-called "cold weld." The adhesion due to this weld is so high that rather than separating at the interface between Au2 and Au3, the thinner Au2 film ruptures, transferring the gold in the contact zone from Au2 to Au3 as indicated by the interference fringes recorded after separation shown in (b), and the recorded optical microscopy images shown in (c) and (d). The scale bar is $100 \mu \mathrm{m}$. 

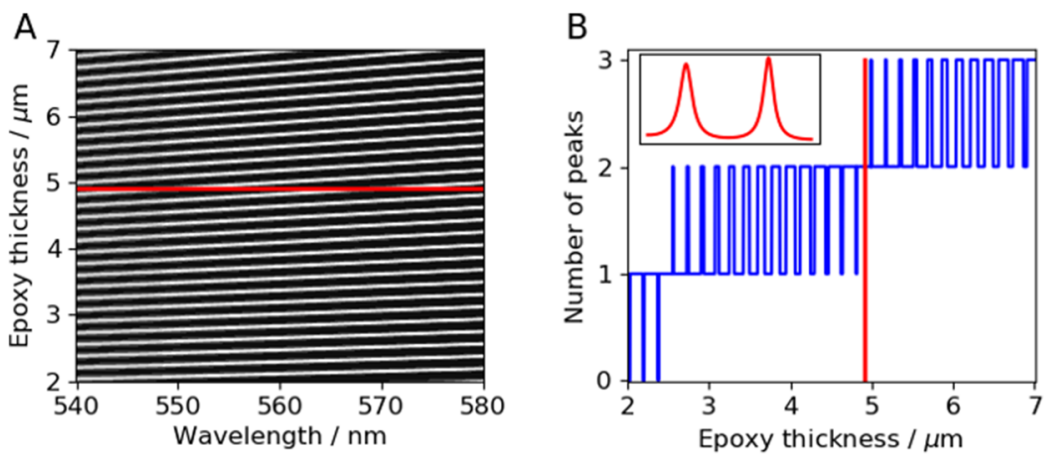

\begin{abstract}
FIG. 9. Optimal thickness of the epoxy spacer layer, using $\mathrm{n}_{\text {spacer }}=1.55, \mathbf{A u 1}=30 \mathrm{~nm}, \mathbf{A u 2}=20 \mathrm{~nm}$, and $\mathbf{A u 3}=30 \mathrm{~nm}$. (a) Intensity plot showing the contact fringe position $(\mathbf{D}=0)$ as a function of epoxy thickness and wavelength. (b) Number of peaks present in the $540-580 \mathrm{~nm}$ wavelength range as a function of epoxy thickness. The red line indicates $\mathbf{Z 1}=4.9 \mu \mathrm{m}$. The inset shows the corresponding interference spectrum.
\end{abstract}

\section{APPENDIX: ADHESION AND COLD WELDING BETWEEN GOLD SURFACES IN DRY GAS ATMOSPHERE}

When pristine metal surfaces are brought into contact in dry gas environment, they can form a so-called "cold weld." Although this does not impact measurements in liquids, for which the technique is principally designed, it does occur in the dry gas experiment used as a proof-of-concept in this work. For this reason, we now briefly describe the observation and interpretation of the cold weld.

In our experiments, gold is evaporated onto freshly cleaved, single-crystalline mica templates. The mica is removed by template stripping in particulate-free laboratory air right before the experiment, with the aim to keep the gold surfaces free of contaminants. After the gold surfaces (Au2, Au3) are brought into contact in our proof-of-principle experiments, they are separated by very slowly pulling the surfaces apart against the deflection of a spring with known spring constant. In principle, the energy of adhesion between the surfaces can be calculated using JKR mechanics, but the rupture of the gold film at the periphery of the contact zone invalidates the use of this model (Fig. 8). However, the "effective adhesion energy" can be used as qualitative probe to estimate the cleanliness of the gold surfaces since adhesion energy between like solids tends to decrease due to the adsorption of contaminants.

The effective adhesion force ( $\left.\mathrm{F}_{\text {Adh,eff }}\right)$ associated with the surface separation after jumping out of contact $(\sigma)$ is equal to $\mathrm{F}_{\mathrm{Adh}, \text { eff }}=\sigma \mathrm{k}$, where $\mathrm{k}$ is the spring constant. The adhesion force for a spherical probe on a flat surface can be related to the adhesion energy as $E_{\text {Adh }}=\left(2 F_{A d h}\right) /(3 \pi R)$, where $\mathrm{R}$ is the radius of curvature. Using $\mathrm{F}_{\mathrm{Adh}}=\mathrm{F}_{\mathrm{Adh}, \mathrm{eff}}$, $\sigma=1 \mathrm{~mm}, \mathrm{k}=124 \mathrm{~N} / \mathrm{m}$, and $\mathrm{R}=10 \mathrm{~mm}, \mathrm{E}_{\mathrm{Adh}} \approx 2.6 \mathrm{~J} / \mathrm{m}^{2}$. This indicates that the surfaces are relatively free of contaminants that may adsorb to the surfaces during the preparation procedure.
${ }^{1}$ R. M. Pashley, J. Colloid Interface Sci. 83(2), 531-546 (1981).

${ }^{2}$ J. N. Israelachvili and G. E. Adams, Nature 262(5571), 774-776 (1976).

${ }^{3}$ U. Raviv and J. Klein, Science 297, 1540-1544 (2002).

${ }^{4}$ A. M. Smith, K. R. J. Lovelock, N. N. Gosvami, T. Welton, and S. Perkin, Phys. Chem. Chem. Phys. 15, 15317-15320 (2013).

${ }^{5}$ A. M. Smith, A. A. Lee, and S. Perkin, J. Phys. Chem. Lett. 7(12), 2157-2163 (2016).

${ }^{6}$ H. K. Christenson and N. H. Thomson, Surf. Sci. Rep. 71(2), 367-390 (2016).

${ }^{7}$ J. N. Israelachvili, J. Colloid Interface Sci. 44(2), 259-272 (1973).

${ }^{8}$ D. F. Kienle and T. L. Kuhl, Anal. Chem. 86, 11860-11867 (2014).

${ }^{9}$ J. J. Blackstock, Z. Li, M. R. Freeman, and D. R. Stewart, Surf. Sci. 546, 87-96 (2003).

${ }^{10}$ L. Chai and J. Klein, Langmuir 23, 7777-7783 (2007).

${ }^{11}$ C. H. Lui, L. Liu, K. F. Mak, G. W. Flynn, and T. F. Heinz, Nature 462 , 339-341 (2009).

${ }^{12}$ S. Fujii, M. Kasuya, and K. Kurihara, J. Phys. Chem. C 121(47), 26406-26413 (2017).

${ }^{13}$ J. Fréchette and T. Kyle Vanderlick, Langmuir 17(24), 7620-7627 (2001).

${ }^{14}$ M. Valtiner, X. Banquy, K. Kristiansen, G. W. Greene, and J. N. Israelachvili, Langmuir 28(36), 13080-13093 (2012).

${ }^{15}$ R. Tivony, D. B. Yaakov, G. Silbert, and J. Klein, Langmuir 31(47), 12845-12849 (2015).

${ }^{16}$ J. N. Connor and R. G. Horn, Rev. Sci. Instrum. 74, 4601-4606 (2003).

${ }^{17}$ B. Moeremans, H.-W. Cheng, Q. Hu, H. F. Garces, N. P. Padture, F. U. Renner, and M. Valtiner, Nat. Commun. 7, 12693 (2016).

${ }^{18}$ J. Britton, N. E. A. Cousens, S. W. Coles, C. D. van Engers, V. Babenko, A. T. Murdock, A. Koós, S. Perkin, and N. Grobert, Langmuir 30(38), 11485-11492 (2014).

${ }^{19}$ J. M. Levins and T. K. Vanderlick, Langmuir 10(7), 2389-2394 (1994).

${ }^{20}$ H. Kawai, H. Sakuma, M. Mizukami, T. Abe, Y. Fukao, H. Tajima, and K. Kurihara, Rev. Sci. Instrum. 79, 043701 (2008).

${ }^{21}$ M. Kasuya and K. Kurihara, Electrochemistry 82, 317-321 (2014).

${ }^{22}$ C. D. Van Engers, N. Cousens, V. Babenko, J. Britton, B. Zappone, N. Grobert, and S. Perkin, Nano Lett. 17(6), 3815-3821 (2017).

${ }^{23}$ M. T. Clarkson, J. Phys. D: Appl. Phys. 22, 475 (1989).

${ }^{24}$ M. Balabajew, C. D. van Engers, and S. Perkin, Rev. Sci. Instrum. 88(12), 123903 (2017).

${ }^{25}$ M. Heuberger, Rev. Sci. Instrum. 72(3), 1700 (2001).

${ }^{26}$ M. Heuberger, G. Luengo, and J. Israelachvili, Langmuir 13(14), 3839-3848 (1997).

${ }^{27}$ J. Klein and E. Kumacheva, J. Chem. Phys. 108(16), 6996 (1998).

${ }^{28}$ T. E. Oliphant, A Guide to NumPy (Trelgol Publishing, USA, 2015).

${ }^{29}$ K. L. Johnson, K. Kendall, and A. D. Roberts, Proc. R. Soc. A 324(1558), 301-313 (1971). 